\title{
Pós-Graduação em Jornalismo no Brasil e 10 anos do PosJor-UFSC
}
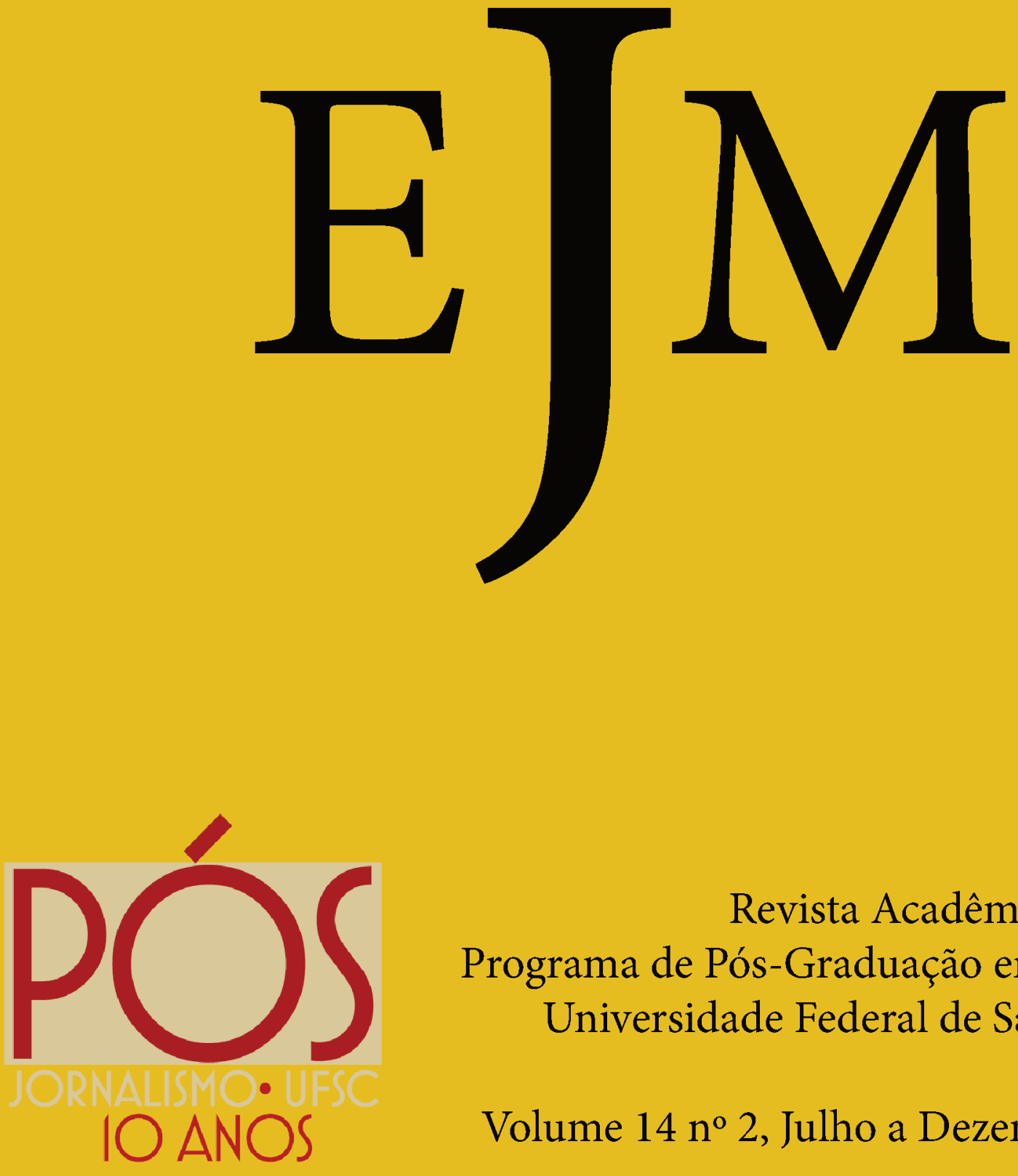

Revista Acadêmica Semestral Programa de Pós-Graduação em Jornalismo Universidade Federal de Santa Catarina Volume $14 \mathrm{n}^{\circ}$ 2, Julho a Dezembro de 2017 


\section{Universidade Federal de Santa Catarina}

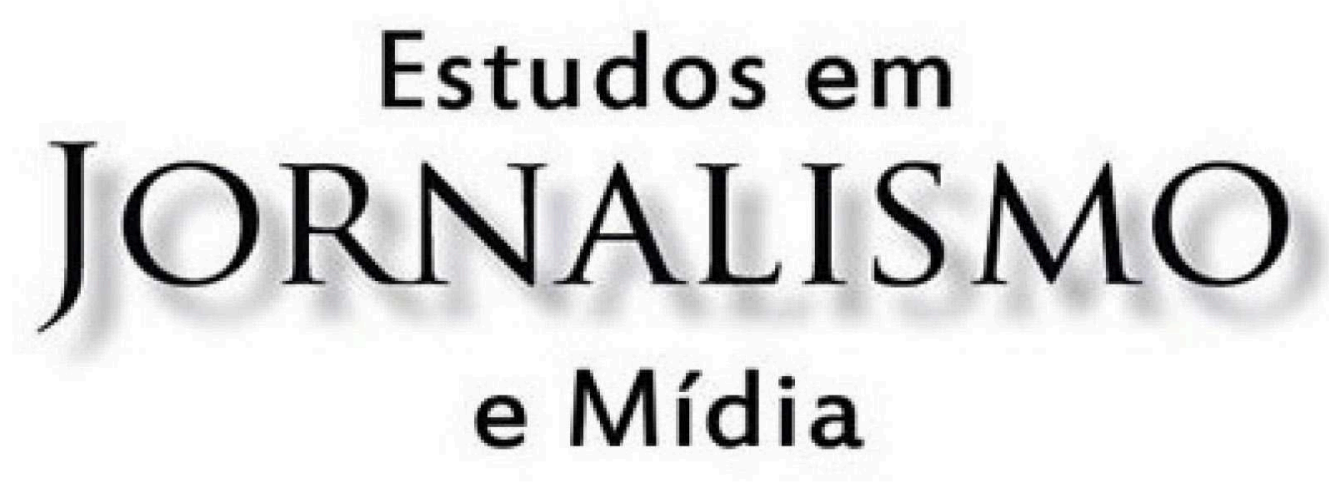

Volume 14 - № 2

Julho a Dezembro 2017

ISSNe 1984-6924

(c) $($ ) $9 \Theta$ 


\title{
Universidade Federal de Santa Catarina
}

\author{
Reitor \\ Ubaldo César Balthazar \\ Pró-Reitor de Pesquisa \\ Sebastião Roberto Soares \\ Pró-Reitor de Pós-Graduação \\ Hugo Moreira Soares \\ Centro de Comunicação e Expressão \\ Diretor \\ Arnoldo Debatin Neto \\ Departamento de Jornalismo \\ Chefe \\ Maria José Baldessar \\ Programa de Pós-Graduação em Jornalismo \\ Coordenadora \\ Raquel Ritter Longhi
}

Estudos em Jornalismo e Mídia - Volume 14, n 2, Julho a Dezembro de 2017

Semestral

Revista Acadêmica Semestral On-line - Programa de Pós-Graduação em Jornalismo da Universidade Federal de Santa Catarina

$2^{\text {a }}$ edição

ISSNe 1984-6924

1. Jornalismo 2. Mídia I.Título

Universidade Federal de Santa Catarina

Programa de Pós-Graduação em Jornalismo

http://www.posjor.ufsc.br

Florianópolis - SC - Brasil

Fone: +55 48 3721-6610

Revista Estudos em Jornalismo e Mídia

http://periodicos.ufsc.br/index.php/jornalismo

E-mail: ejm@contato.ufsc.br 


\section{Estudos em \\ JORNALISMO \\ e Mídia}

Comissão Editorial: Jorge Kanehide Ijuim e Daiane Bertasso Ribeiro (editores)

Projeto Gráfico e Capa: Sílvio da Costa Pereira e Ana Carolina Passos

Entrevistadora: Lívia de Souza Vieira

Revisão: Ana Marta Moreira Flores e Amanda Miranda (traduções),

Criselli Maria Montipó, Dairan Paul, Daniela Caniçali Martins, Edwin Carvalho,

Géssica Gabrieli Valentini, Hendryo Anderson André, Ingrid Pereira De Assis, Janaíne

Kronbauer dos Santos, Janara Nicoletti, Juliana Gobbi Betti, Kérley Winques, Magali

Moser, Rafael Rangel Winch, Ricardo José Torres, William Robson Cordeiro Silva.

Editoração e produção: Ana Carolina Passos

Apoio de Produção: Sílvio da Costa Pereira e Equipe do Portal de Periódicos da UFSC

\section{Conselho Editorial}

Adriana Amado, UNLAM, Argentina

Aglair Bernardo, UFSC, Brasil

Alfredo Vizeu, UFPE, Brasil

Antonio Hohlfeldt, PUC-RS, Brasil

Ana Carolina Escosteguy, PUC-RS, Brasil

Ana Paula Goulart Ribeiro, UFRJ, Brasil

Basilio G. Monteiro, Saint John's University, EUA

Beatriz Becker, UFRJ, Brasil

Carlos Eduardo Franciscato, UFS, Brasil

Claudia Lago, ECA/USP, Brasil

Claudia Mellado Ruiz, Usach, Chile

Christa Berger, Unisinos, Brasil

Dione Oliveira Moura, UnB, Brasil

Dóris Fagundes Haussen, PUC-RS, Brasil

Edson Fernando Dalmonte, UFBA, Brasil

Eduardo Meditsch, UFSC, Brasil

Elias Machado Gonçalves, UFSC, Brasil

Elton Antunes, UFMG, Brasil

Flávio Porcello, UFRGS, Brasil

Francisco José Castilhos Karam, UFSC, Brasil

Gislene Silva, UFSC, Brasil

Heloíza Herscovitz, California State Univ., EUA

Isabel Ferin Cunha, U. Coimbra, Portugal
Jacques Mick, UFSC, Brasil

João Canavilhas, UBI, Portugal

Jorge Pedro Sousa, Un. Fernando Pessoa, Portugal

José Marques de Melo, Umesp, Brasil

Josenildo Luiz Guerra, UFS, Brasil

Juan Carlos Suárez Villegas, Un. Sevilla, Espanha

Kênia Beatriz Ferreira Maia, UFRN, Brasil

Lila Luchessi, UNRN, Argentina

Luciana Mielniczuk, UFRGS, Brasil

Luiz Gonzaga Motta, UnB-UFSC, Brasil

Manuel Pinto, Universidade do Minho, Portugal

Marcos Palácios, UFBA, Brasil

Marcia Benetti, UFRGS, Brasil

Maria José Baldessar, UFSC, Brasil

Martín Becerra, UBA, Argentina

Nilson Lemos Lage, UFSC, Brasil

Paulo Bernardo Ferreira Vaz, UFMG, Brasil

Rosana Soares, USP, Brasil

Silvio Waisbord, George Washington Univ., EUA

Sonia Virgínia Moreira, UERJ, Brasil

Susana Herrera Damas, Univ. Carlos III, Espanha

Suzana Barbosa, UFBA, Brasil

Victor Gentilli, UFES, Brasil 